\title{
Psychological Distress and Weight Gain in Pregnancy: a Population-Based Study
}

\author{
Florianne O. L. Vehmeijer ${ }^{1,2} \cdot$ Sangeeta R. Balkaran ${ }^{1,2} \cdot$ Susana Santos $^{1,3} \cdot$ Romy Gaillard $^{1,3} \cdot$ Janine F. Felix ${ }^{1,2}$. \\ Manon H. J. Hillegers ${ }^{2,4}$. Hanan El Marroun ${ }^{2,4}$ - Vincent W. V. Jaddoe ${ }^{1,3}$
}

Published online: 18 December 2019

(C) The Author(s) 2019

\begin{abstract}
Background Psychological distress and inappropriate or excessive weight gain are common in pregnancy and are associated with adverse maternal and offspring outcomes. Psychological well-being and weight status of women during pregnancy might be interrelated. We aimed to examine whether psychological distress during pregnancy is associated with gestational weight gain. Method In a population-based cohort of 3393 pregnant women, information about psychological distress, depressive and anxiety symptoms was assessed at 20 weeks of gestation using the Brief Symptom Inventory questionnaire. Weight was repeatedly measured during pregnancy and obtained by questionnaire before and after pregnancy. Linear regression and multinomial logistic regression models were used. Weight gain in the second half of pregnancy, total weight gain, and the risks of inadequate and excessive total weight gain were the main outcome measures.

Results In total, 7.0\% of all women experienced psychological distress. Overall psychological distress and anxiety were associated with lower weight gain in the second half of pregnancy (differences $-1.00 \mathrm{~kg}(95 \%$ confidence interval (CI) - 1.62, $0.37)$ and $-0.68 \mathrm{~kg}(95 \% \mathrm{CI}-1.24,-0.11)$, respectively). These associations fully attenuated into non-significance after taking account for socio-demographic variables. Similar results were observed for total weight gain. Only women with anxiety symptoms had, independently of potential confounders, a lower risk of excessive weight gain (odds ratio (OR) 0.61 (95\% CI 0.48 , $0.91)$ ).

Conclusions In this large prospective cohort study, the observed associations of psychological distress with weight gain during pregnancy seem to be largely explained by common socio-demographic factors.
\end{abstract}

Keywords Psychological distress $\cdot$ Depression, anxiety $\cdot$ Pregnancy $\cdot$ Gestational weight gain

Electronic supplementary material The online version of this article (https://doi.org/10.1007/s12529-019-09832-0) contains supplementary material, which is available to authorized users.

Vincent W. V. Jaddoe

v.jaddoe@erasmusmc.nl

1 The Generation R Study Group, Erasmus MC, University Medical Center, Rotterdam, The Netherlands

2 Department of Epidemiology, Erasmus MC, University Medical Center, Rotterdam, The Netherlands

3 Department of Pediatrics, Erasmus MC, University Medical Center, Rotterdam, The Netherlands

4 Department of Child and Adolescent Psychiatry/Psychology, Erasmus MC, University Medical Center, Rotterdam, The Netherlands

\section{Introduction}

Psychological distress is generally defined as general stress, depressive symptoms, anxiety or experiencing an adverse life event $[1,2]$. In western countries, 5-20\% of pregnant women experience psychological distress [2-4]. Also, more than 60\% of pregnant women have either inadequate or excessive weight gain [5, 6]. Pregnancy is a critical period for psychological distress and weight gain, since both are associated with increased risks of adverse maternal and offspring outcomes [7-11]. Previously, we have reported that, compared with low or recommended weight gain, excessive weight gain was associated with a higher risk of gestational hypertension, cesarean delivery, large size for gestational age infants, and childhood overweight [12]. We have also reported that anxiety and depression during pregnancy were associated with impaired fetal growth [13]. Psychological distress and weight 
gain in pregnant women may also affect each other [14-17]. Two systematic reviews among, in total, 12 studies have been performed on the association between psychological distress and weight gain in pregnancy. One systematic review showed no association and the second systematic review only reported an association of depression, but not psychological distress and anxiety, with increased gestational weight gain [7, 9]. These reviews compiled studies with a modest sample size, used different definitions of psychological distress, depression and anxiety and did not define cutoffs for psychological distress to consider clinical importance.

We hypothesized that psychological distress during pregnancy is associated with gestational weight gain. We examined in a population-based prospective cohort study among 3393 pregnant women the associations of psychological distress during pregnancy and gestational weight gain. We also explored whether any association was explained by sociodemographic or lifestyle-related variables.

\section{Methods}

\section{Study Design}

This study was embedded in the Generation R Study, a population-based prospective cohort study from early pregnancy until young adulthood in Rotterdam, the Netherlands $[18,19]$. The study was approved by the Medical Ethics Committee of Erasmus Medical Center Rotterdam, The Netherlands (MEC 198.782/2001/31). Pregnant women were enrolled between 2002 and 2006. Written informed consent was obtained from all women in the study. In total, 8879 mothers (91\% of the full cohort) were enrolled during pregnancy of whom information about psychological distress during pregnancy was available in 6650 . We further excluded pregnancies not leading to singleton live births $(N=101)$ and women without information on weight gain during pregnancy $(N=3156)$. This resulted in a population for analysis of 3393 mothers.

\section{Psychological Distress During Pregnancy}

The Brief Symptom Inventory (BSI) questionnaire was used to examine psychological distress at approximately 20 weeks of gestation. The BSI is a validated self-report questionnaire consisting of 53 items, describing multidimensional psychopathologic problems and complaints in adults in the preceding 7 days [20-23]. The items are divided in 9 subscales (including anxiety, depression, hostility, phobic anxiety, interpersonal sensitivity, obsessive-compulsiveness, paranoid ideation, psychoticism, somatization). As an indicator of overall psychological distress, we used the Global Severity Index (GSI) that is a total score of the 9 subscales (all 53 items of the BSI).
Additionally, we used the depression and anxiety subscales separately. We chose these 2 subscales because they are widely used as valid proxies for psychological distress during pregnancy $[1,2]$. The items were rated on a 5 -point unidimensional scale ranging from " 0 " (not at all) to " 4 " (extremely). A total score was calculated for each symptom scale by summing the item scores of the scales and dividing the results by the number of items in that scale. Higher scores represented an increased occurrence of symptoms. Psychological symptoms were dichotomized (yes/no) by using the following clinical cutoffs derived from a psychiatric outpatient sample of Dutch non-pregnant women: 0.71 for overall psychological symptoms scale, 0.80 for the depression symptoms scale and 0.71 for the anxiety symptoms scale [23].

\section{Weight Measurements During Pregnancy}

As enrolment in our study was in pregnancy, we were not able to measure weight before pregnancy. Information on prepregnancy weight was obtained by questionnaires at enrollment. Pre-pregnancy body mass index (BMI) in $\mathrm{kg} / \mathrm{m}^{2}$ was calculated using pre-pregnancy weight $(\mathrm{kg})$ as reported by the mother and height $(\mathrm{m})$ measured at enrolment and was categorized into underweight $\left(<18.5 \mathrm{~kg} / \mathrm{m}^{2}\right)$, normal weight $\left(18.5-24.9 \mathrm{~kg} / \mathrm{m}^{2}\right)$, overweight $\left(25-29.9 \mathrm{~kg} / \mathrm{m}^{2}\right)$, and obesity $\left(\geq 30 \mathrm{~kg} / \mathrm{m}^{2}\right.$ ) according to the World Health Organization (WHO) 2000 criteria [24]. Weight measurements during pregnancy were performed in a dedicated research center without shoes and heavy clothing. Since we collected information about psychological distress at 20 weeks, we used weight gain in the second half of pregnancy as main outcome. Weight gain in the second half of pregnancy was calculated subtracting weight measured in mid-pregnancy (median 20.4 weeks (95\% range 18.6-24.9 weeks)) from the maximum reported weight in pregnancy. The latest weight before delivery was obtained by questionnaire completed 2 months after delivery, hereafter referred to as maximum reported weight in pregnancy. Total weight gain during pregnancy was calculated by subtracting reported pre-pregnancy weight from the maximum reported weight in pregnancy. Total gestational weight gain was categorized into inadequate, adequate, and excessive weight gain following the 2009 Institute of Medicine (IOM) weight gain recommendation criteria using both categorized pre-pregnancy BMI and total gestational weight gain [25-27].

\section{Covariates}

Gestational age was established using the last menstrual period or first trimester ultrasound measurement [28]. We obtained information on maternal age, ethnicity, parity, educational level, and marital status by questionnaire at enrolment [18]. Information about folic acid intake, smoking, alcohol 
consumption and nutritional intake in kcal (kilocalories) was assessed by questionnaires during pregnancy.

\section{Statistical Analysis}

First, we compared subject characteristics between women with and those without psychological distress using Pearson's chi-square tests, independent sample $t$ tests and Mann-Whitney tests. We performed non-response analysis using the same tests to assess differences between women with and without information on weight gain during pregnancy. We compared psychological distress and weight gain characteristics between pre-pregnancy BMI categories. Second, we used linear regression models to assess the associations of overall psychological distress, depression, and anxiety with weight gain in the second half of pregnancy and total gestational weight gain. We examined potential interactions of maternal psychological distress with pre-pregnancy BMI and ethnicity in the association with gestational weight gain. We observed a statistically significant interaction of maternal psychological distress and pre-pregnancy BMI and thus performed stratified analyses for the clinical BMI categories according to the WHO 2000 criteria (underweight, normal weight, overweight, obesity). Further, we performed sensitivity analyses among full-term mothers, defined as a gestational age of $\geq 37$ weeks at birth, to exclude potential bias by preterm birth. Third, we used multinomial logistic regression models to assess the associations of psychological distress, depression, and anxiety with clinical categories of gestational weight gain according to the IOM criteria (inadequate, adequate and excessive weight gain). For all regression models, the basic models were adjusted for maternal age, whereas the full models were adjusted for potential confounders. We included covariates in the models if they were associated with psychological distress and gestational weight gain or if they changed the effect estimates substantially ( $>10 \%)$. In order to maintain statistical power and reduce bias related to missing data on covariates (missing data on covariates ranged from 0 to $21 \%$, see Electronic Supplementary Material 2), we performed multiple imputation using the Markov Chain Monte Carlo method. Exploratory analyses showed the data was not missing completely at random (MCAR) (indicated by the Little's test, $p$ value <0.05) [29]. Comparison between characteristics of complete cases (participants with no missing data) and participants with at least one missing value showed no large differences. Considering these results and no likely reason for the data to be missing not at random (MNAR), we proceeded with multiple imputation for which missing at random (MAR) is an assumption [30]. Five new datasets were created and pooled results are presented. No major differences in descriptive statistics were found between the original and imputed datasets. Statistical analyses were performed with the Statistical
Package of Social Sciences version 21.0 for Windows (IBM Corp, Armonk, NY, USA).

\section{Results}

\section{Subject Characteristics}

Psychological distress, depression, and anxiety were reported by $7.0 \%, 7.0 \%$, and $8.4 \%$ of all pregnant women, respectively. Table 1 shows the subject characteristics. In total, $20.1 \%$ and $45.0 \%$ of all women had inadequate and excessive gestational weight gain, respectively. Compared to women without psychological distress, those with psychological distress were more often younger, lower educated, without a partner, of a non-Dutch ethnicity, continued smokers, and had a lower nutritional intake, alcohol, and folic acid supplement use (Table 1). Table 2 shows that the prevalence of psychological distress, depression, and anxiety varies between BMI categories. Women with a normal pre-pregnancy weight had the lowest prevalence of psychological distress and the highest mean weight gain.

Non-response analyses showed that, as compared to women with missing information on weight gain during pregnancy, those with information on gestational weight gain had a higher maternal age, a lower pre-pregnancy BMI, a partner, a higher intake of alcohol and folic acid and a higher total daily energy intake, and were more often nulliparous, higher educated and Dutch-European (Electronic Supplementary Material 2).

\section{Psychological Distress and Weight Gain in the Second Half of Pregnancy}

Figure 1 shows that, in the basic models, overall psychological distress and anxiety were associated with lower weight gain in the second half of pregnancy (differences $-1.00 \mathrm{~kg}(95 \%$ confidence interval $(\mathrm{CI})-1.62,-0.37)$ and $-0.68 \mathrm{~kg}(95 \%$ CI $-1.24,-0.11$ ), for overall psychological distress or anxiety, respectively). These associations fully attenuated into nonsignificance in the adjusted model after taking account for socio-demographic variables such as maternal education and ethnicity. Maternal depression during pregnancy was not associated with weight gain in the second half of pregnancy.

For the associations of maternal psychological distress, depression and anxiety with total gestational weight gain, effect estimates were of the same magnitude and in similar direction as for weight gain in the second half of pregnancy (Electronic Supplementary Material 3). The stratified analyses for different BMI categories are shown in Electronic Supplementary Material 4. Underweight women experiencing overall psychological distress or depression tended to have an increased weight gain from 20 weeks onwards compared with normal, overweight, and obese women with overall psychological distress or depression; however, results were not significant. In obese women 
Table 1 Characteristics of study population $(N=3393)^{\text {a }}$

\begin{tabular}{|c|c|c|c|c|}
\hline & $\begin{array}{l}\text { Population for } \\
\text { analysis }(N=3393)\end{array}$ & $\begin{array}{l}\text { Psychological } \\
\text { distress }(N=238)\end{array}$ & $\begin{array}{l}\text { No psychological } \\
\text { distress }(N=3155)\end{array}$ & $P$-value ${ }^{\mathrm{b}}$ \\
\hline Age at intake, mean (SD), years & $31.0(4.7)$ & $28.2(5.8)$ & $31.3(4.5)$ & $<0.001$ \\
\hline Pre-pregnancy weight, median (95\% range), $\mathrm{kg}$ & $64.0(49.0,97.0)$ & $62.0(47.0,104.6)$ & $64.0(49.9,96.2)$ & $<0.01$ \\
\hline Pre-pregnancy BMI, median (95\% range) $\mathrm{kg} / \mathrm{m}^{2}$ & $22.3(18.2,33.6)$ & $22.7(17.9,36.7)$ & $22.3(18.2,33.3)$ & $<0.01$ \\
\hline Pre-pregnancy BMI clinical categories, $N(\%)$ & & & & $<0.01$ \\
\hline Underweight & $111(3.8)$ & $9(4.4)$ & $102(3.8)$ & \\
\hline Normal weight & $2127(73.0)$ & $140(68.6)$ & $1987(73.3)$ & \\
\hline Overweight & $491(16.8)$ & $40(19.6)$ & $451(16.6)$ & \\
\hline Obesity & $185(6.3)$ & $15(7.4)$ & $170(6.3)$ & \\
\hline Gestational age at birth, median (95\% range), weeks & $40.1(36.3,42.4)$ & $40.3(36.1,42.6)$ & $40.0(36.3,42.4)$ & 0.21 \\
\hline Total weight gain, mean (SD), kg & $14.9(5.9)$ & $14.4(6.7)$ & $15.0(5.8)$ & 0.17 \\
\hline Gestational weight gain clinical categories (IOM criteria), $N(\%)$ & & & & 0.21 \\
\hline Inadequate weight gain & $586(20.1)$ & $49(24.0)$ & $537(19.8)$ & \\
\hline Adequate weight gain & $1018(34.9)$ & $74(36.3)$ & $944(34.8)$ & \\
\hline Excessive weight gain & $1310(45.0)$ & $81(39.7)$ & $1229(45.4)$ & \\
\hline Weight gain 2nd half of pregnancy, mean(SD), $\mathrm{kg}$ & $9.6(4.6)$ & $9.0(4.8)$ & $9.6(4.6)$ & \\
\hline Parity, $N(\%)$ & & & & 0.23 \\
\hline Nulliparous & $2059(60.7)$ & $144(60.5)$ & $1915(60.7)$ & \\
\hline Multiparous & $1334(39.7)$ & $94(39.5)$ & $1240(39.3)$ & \\
\hline Education, $N(\%)$ & & & & $<0.001$ \\
\hline Primary school & $178(5.3)$ & $31(13.0)$ & $147(4.7)$ & \\
\hline Secondary school & $1280(37.7)$ & $138(58.0)$ & $1142(36.2)$ & \\
\hline Higher education & $1935(57.0)$ & $69(29.0)$ & $1866(59.1)$ & \\
\hline Marital status, $N(\%)$ & & & & $<0.001$ \\
\hline Married/living together & $3084(90.9)$ & $185(77.7)$ & $2899(91.9)$ & \\
\hline No partner & $309(9.1)$ & $53(22.3)$ & $256(8.1)$ & \\
\hline Ethnicity, $N(\%)$ & & & & $<0.001$ \\
\hline Dutch-European & $2448(72.1)$ & $92(38.6)$ & $2356(74.6)$ & \\
\hline Surinamese & $200(5.9)$ & $28(11.8)$ & $172(5.5)$ & \\
\hline Turkish & $178(5.2)$ & $46(19.3)$ & $132(4.2)$ & \\
\hline Moroccan & $109(3.2)$ & $24(10.1)$ & $85(2.7)$ & \\
\hline Cape Verdian & $70(2.1)$ & $19(8.0)$ & $52(1.6)$ & \\
\hline Dutch Antilles & $69(2.1)$ & $6(2.5)$ & $62(2.0)$ & \\
\hline Others & $319(9.4)$ & $23(9.7)$ & $296(9.4)$ & \\
\hline Alcohol consumption, $N(\%)$ & & & & $<0.001$ \\
\hline No & $1304(38.4)$ & $130(54.6)$ & $1173(37.2)$ & \\
\hline Yes & $2089(61.6)$ & $108(45.4)$ & $1982(62.8)$ & \\
\hline Smoking habits, $N(\%)$ & & & & $<0.001$ \\
\hline No & $2584(76.2)$ & $145(60.9)$ & $2439(77.3)$ & \\
\hline During first trimester only & $318(9.3)$ & $23(9.7)$ & $295(9.4)$ & \\
\hline Continued during pregnancy & $491(14.5)$ & $70(29.4)$ & $421(13.3)$ & \\
\hline Folic acid supplement use, $N(\%)$ & & & & $<0.001$ \\
\hline No & $627(18.5)$ & $89(37.4)$ & $538(17.1)$ & \\
\hline Start during first 10 weeks of pregnancy & $1109(32.7)$ & $102(42.9)$ & $1007(31.9)$ & \\
\hline Preconception use & $1657(48.8)$ & 47 (19.7) & $1610(51.0)$ & \\
\hline Total daily energy intake, mean (SD), kcal & $2076(535)$ & $2015(529)$ & $2081(530)$ & $<0.01$ \\
\hline
\end{tabular}

${ }^{a}$ Values are means (standard deviation) for continuous variables with a normal distribution, or medians (95\% range) for continuous variables with a skewed distribution, and valid percentages for categorical variables. Missing values in covariates are imputed. Percentages of pre-pregnancy BMI clinical categories and gestational weight gain clinical categories are valid percentages

${ }^{\mathrm{b}} P$-values for differences in subject characteristics between mothers with psychological distress and mothers without psychological distress were calculated performing independent sample $t$ tests for normally distributed continuous variables, the Mann-Whitney test for not normally distributed continues variables and chi-square tests for categorical variables 
Table 2 Psychological distress and gestational weight gain characteristics by pre-pregnancy BMI category $(N=3393)^{\mathrm{a}}$

\begin{tabular}{|c|c|c|c|c|}
\hline & \multicolumn{4}{|c|}{ Pre-pregnancy BMI categories } \\
\hline & $\begin{array}{l}\text { Underweight } \\
N=111\end{array}$ & $\begin{array}{l}\text { Normal weight } \\
N=2127\end{array}$ & $\begin{array}{l}\text { Overweight } \\
N=491\end{array}$ & $\begin{array}{l}\text { Obesity } \\
N=185\end{array}$ \\
\hline \multicolumn{5}{|l|}{ Weight gain measurements } \\
\hline Total gestational weight gain, mean (SD) & $14.4(5.3)$ & $15.4(5.3)$ & $14.3(6.4)$ & $11.5(8.6)$ \\
\hline Weight gain in the second half of pregnancy, mean (SD) & $9.1(4.5)$ & $9.8(4.3)$ & $9.5(4.8)$ & $8.0(5.7)$ \\
\hline \multicolumn{5}{|l|}{ Psychological measurements } \\
\hline Psychological distress & $9(8.1 \%)$ & $140(6.6 \%)$ & $40(8.1 \%)$ & $15(8.1 \%)$ \\
\hline No psychological distress & $102(91.9 \%)$ & $1987(93.4 \%)$ & $451(91.9 \%)$ & $170(91.9 \%)$ \\
\hline Depression & $10(9.0 \%)$ & $134(6.3 \%)$ & $41(8.4 \%)$ & $10(5.4 \%)$ \\
\hline No depression & $101(91.0 \%)$ & $1992(93.7 \%)$ & $450(91.6 \%)$ & $174(94.1 \%)$ \\
\hline Anxiety & $8(7.2 \%)$ & $173(8.1 \%)$ & $50(10.2 \%)$ & $16(8.6 \%)$ \\
\hline No anxiety & $103(92.8 \%)$ & $1954(91.9 \%)$ & $441(89.9 \%)$ & $169(91.4 \%)$ \\
\hline
\end{tabular}

experiencing anxiety during pregnancy, weight gain in the second half of pregnancy tended to be lower. Sensitivity analyses among women who had a full term pregnancy only, showed effect estimates of the same size and direction as the main analyses (Electronic Supplementary Material 5).

\section{Psychological Distress and the Risk of Inadequate and Excessive Weight Gain in Pregnancy}

Overall psychological distress and depression were not associated with the risks of inadequate or excessive weight gain (Fig. 2). Maternal anxiety during pregnancy was, independently of confounders, associated with a lower risk of excessive weight gain (odds ratio 0.61 (95\% CI 0.48, 0.91) (Fig. 2). Similar results were obtained without adjustment for pre-pregnancy BMI.

\section{Discussion}

\section{Main Findings}

In this population-based prospective cohort study, we did not observe consistent associations of overall psychological distress, depression, and anxiety with gestational weight gain. Most associations were explained by maternal ethnicity and educational level. Only women with anxiety symptoms had, independently of potential confounders, a lower risk of excessive weight gain.

\section{Interpretation of Main Findings}

We observed that $7.0 \%$ to $8.4 \%$ of all pregnant women reported psychological distress, depression, or anxiety. These percentages are comparable or slightly lower compared to the prevalence reported in previous studies [2, 3]. In our study population,
$23.1 \%$ of women had pre-pregnancy overweight or obesity and $20.1 \%$ and $45.0 \%$ of women experienced inadequate and excessive weight gain, respectively, which is in line with population figures [5, 31-34]. Although results of some previous studies suggested that psychological distress, depression, or anxiety during pregnancy are associated with gestational weight gain [14-17], one systematic review has reported no association [9] and a second systematic review only reported an association of depression, but not psychological distress and anxiety, with gestational weight gain [7]. Most previous studies did not define cutoffs for psychological distress to consider clinical importance and sample sizes were modest. In our large population-based prospective cohort study, we observed that overall psychological distress and anxiety were associated with a lower gestational weight gain. However, these associations attenuated after adjustment for socio-demographic factors. These findings are in line with a study among 1605 women in the USA in which the relationship between psychosocial status and adequacy of gestational weight gain was also influenced by sociodemographic factors [31]. In a large study among 13,314 pregnant women in the UK between 1991 and 1992, the association between antenatal depression and inadequate or excessive gestational weight gain was already non-significant in the unadjusted model [35]. However, this study did not assess the association of overall psychological distress and anxiety with gestational weight gain. One previous study in 242 women found that depression during pregnancy was associated with excessive gestational weight gain, only among women with a high pre-pregnancy BMI [16]. We did not find significant differences in the association of psychological distress, depression, or anxiety with weight gain during pregnancy between women of different pre-pregnancy BMI categories. Thus, results from our and some other studies suggest that common socio-demographic factors explain the 


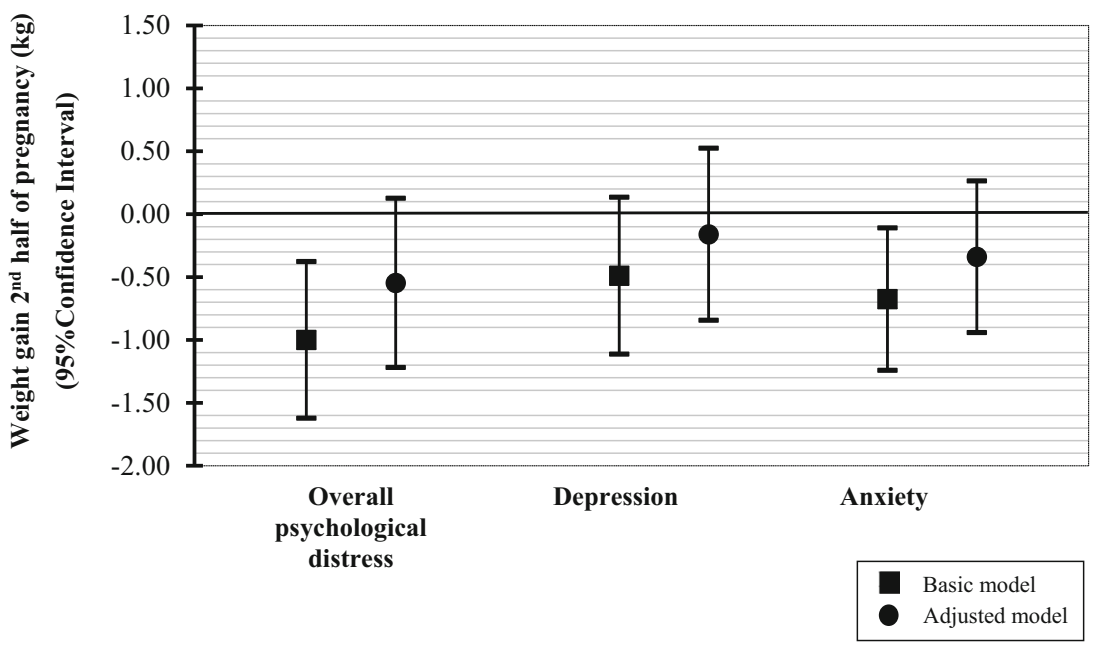

Fig. 1 Associations of psychological distress and weight gain in the second half of pregnancy $(N=3263)$. Values are linear regression coefficients ( $95 \%$ confidence intervals) and represent the overall change in weight gain in the second half of pregnancy for psychological distress, depression and anxiety compared to no psychological distress, depression

association between psychological distress and weight gain during pregnancy.

In the present study, we observed a negative association between anxiety and the risk for excessive weight gain, which remained after adjustment. Our finding is not in line with a recent study among 725 women in which lower reported stress was associated with a greater chance of women achieving adequate gestational weight gain [14]. However, some previous studies also suggest that anxiety and depression may be protective of increased weight gain $[15,17]$. In the USA-study mentioned above, or anxiety. The basic model was adjusted for maternal age. The adjusted model was adjusted for maternal age, pre-pregnancy BMI, parity, education, marital status, ethnicity, alcohol intake, smoking, folic acid use and nutritional intake in kcal

the association between anxiety and a higher adequacy of weight gain disappeared after adjustment for confounders, among which was physical activity [31]. In our study, the association between anxiety and the risk for excessive weight gain remained significant. However, residual confounding by for example physical activity level and sedentary behavior may still be present.

The relationship between psychological distress and weight is complex and might be bidirectional $[7,36,37]$. Since observational studies are not able to clarify the causal directions, mechanistic studies and Mendelian Randomization studies may give
Fig. 2 Associations of psychological distress with clinical categories of gestational weight gain $(N=2914)$. Odds ratios (95\% confidence intervals) represent the risks for the different weight gain categories (inadequate, adequate (reference) and excessive weight gain) according to the 2009 IOM gestational weight gain recommendation categories for women with overall psychological distress, depression and anxiety. The basic model is adjusted for maternal age. The adjusted model is adjusted for maternal age, prepregnancy BMI, parity, educational level, marital status, ethnicity, alcohol intake, smoking, folic acid intake, and nutritional intake in kcal

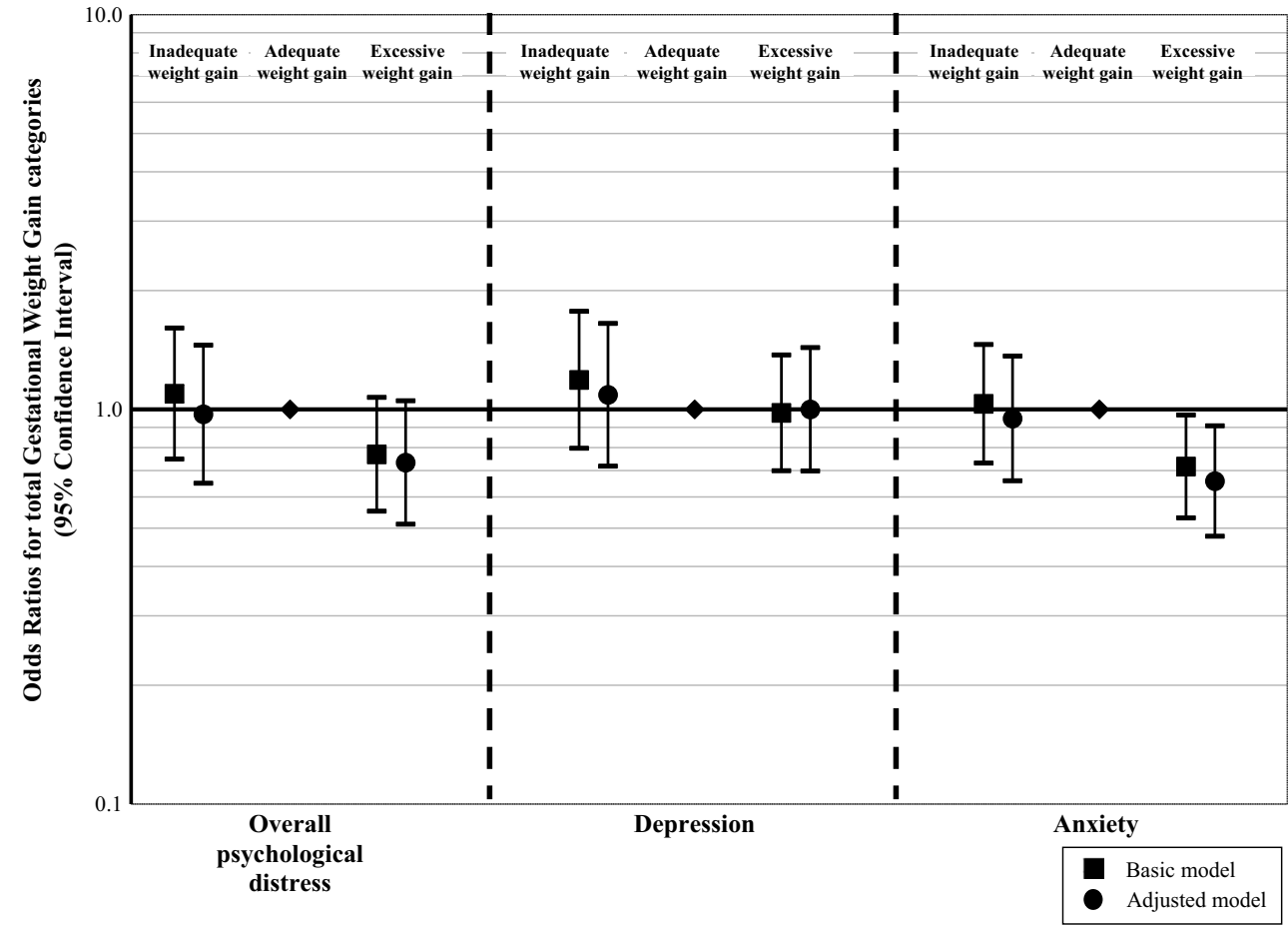


further insight in the underlying mechanisms and directions [38]. This is of great importance because both psychological health and weight gain can be targets for preventive strategies in pregnancy. This is shown in a randomized controlled trial in which the effects of a four-session intervention, motivating participants to have a healthy lifestyle during pregnancy, were examined [39]. The study found a reduction of gestational weight gain and levels of anxiety in obese pregnant women after the intervention.

\section{Strengths and Limitations}

Strengths of this population-based cohort study were the prospective data collection, the detailed measurements from pregnancy onwards and the large sample size of more than 3000 participants. This study also has limitations. Of all women included during pregnancy, $75 \%$ responded to the questionnaire. Only $52 \%$ of all women included during pregnancy with information on psychological distress and with singleton live-born children, had information on weight gain during pregnancy. Non-response could have led to selection bias if the associations were different between those included and not included in the analyses. Extrapolating results to all pregnant women should therefore be done with caution. Women with missing information on psychological distress and weight gain were more often lower educated and of non-European ethnicity. We cannot exclude the possibility that these differences have affected the results. Information on gestational weight gain was self-reported. Self-reported weight tends to be underestimated. We used self-reported weights because the measured weights, weight in the first trimester (median 13.2 weeks, 95\% range 9.8-17.5 weeks) and weight in the third trimester (median 30.2 weeks, 95\% range 28.5-32.6 weeks), do not comprise the whole pregnancy. However, the correlations between self-reported pre-pregnancy weight and measured weight in the first trimester $(r=0.96, P<0.001)$ as well as the correlation between maximum self-reported weight and measured weight in the third trimester were high $(r=0.95$, $P<0.001)$. Psychological distress was measured at only one time point during pregnancy, on average at 20 weeks, and refers to the preceding 7 days. Most other studies also have only one assessment point [40]. Therefore, we do not know whether psychological distress symptoms varied in intensity or were persistent throughout pregnancy. Further research is needed to assess the associations of trimester-specific psychological distress on gestational weight gain. To classify psychological symptoms, we have used cutoffs derived from a clinical population of Dutch nonpregnant women, which might not be entirely suitable for our study population. However, cutoffs from a sample of pregnant women are currently not available. In this study, differential misclassification could occur when women with more psychological distress, depression or anxiety report differently their weight status compared to women without psychological distress, depression, or anxiety. This seems unlikely because both pregnant women and data collectors were unaware of the specific research questions under study [41]. Finally, although we used a large number of confounders, residual confounding might still be present.

\section{Conclusions}

Our results do not support the hypothesis that psychological distress, depression, and anxiety affect weight gain in pregnant women. Only women with anxiety symptoms had, independently of potential confounders, a lower risk of excessive weight gain. The observed associations of psychological distress with weight gain during pregnancy seem to be largely explained by common socio-demographic factors. Further studies are needed to explore whether psychological distress in pregnancy affects other outcomes in women and their children, such as postpartum weight gain/loss.

Acknowledgments We gratefully acknowledge the contribution of the participating children, their mothers, general practitioners, hospitals, midwives and pharmacies in Rotterdam.

Author's Contributions FV and VJ designed and conducted the study. FV and SB analyzed the data and wrote the article. VJ contributed to the interpretation of the data, revisions and gave input at all stages of the study. SS, RG, JF, MH, and HM advised and reviewed the manuscript. All authors have approved the final version of the manuscript.

Funding Information This phase of the Generation R Study was supported by the Erasmus MC, Erasmus University Rotterdam, The Netherlands, Organization for Health Research and Development (ZonMw) and the Ministry of Health, Welfare and Sport. VWVJ received grants from the Netherlands Organization for Health Research and Development (VIDI 016.136.361) and European Research Council (ERC-2014-CoG648916). The funders had no role in the design of the study; the data collection and analyses; the interpretation of data or writing this report.

\section{Compliance with Ethical Standards}

Conflict of Interest The authors declare that they have no conflicts of interest.

Ethical Approval All procedures performed in studies involving human participants were in accordance with the ethical standards of the institutional research committee (Medical Ethics Committee of Erasmus Medical Center Rotterdam, The Netherlands (MEC 198.782/2001/31) and with the 1964 Helsinki declaration and its later amendments or comparable ethical standards.

Consent for Publication Not applicable.

Abbreviations BMI, Body mass index; BSI, Brief symptom inventory; IOM, Institute of Medicine; GSI, Global Severity Index; WHO, World Health Organization

Open Access This article is licensed under a Creative Commons Attribution 4.0 International License, which permits use, sharing, adaptation, distribution and reproduction in any medium or format, as long as you give appropriate credit to the original author(s) and the 
source, provide a link to the Creative Commons licence, and indicate if changes were made. The images or other third party material in this article are included in the article's Creative Commons licence, unless indicated otherwise in a credit line to the material. If material is not included in the article's Creative Commons licence and your intended use is not permitted by statutory regulation or exceeds the permitted use, you will need to obtain permission directly from the copyright holder. To view a copy of this licence, visit http://creativecommons.org/licenses/by/4.0/.

\section{References}

1. Ruiz RJ, Fullerton JT. The measurement of stress in pregnancy. Nurs Health Sci. 1999;1:19-25.

2. Woods SM, Melville JL, Guo Y, Fan M-Y, Gavin A. Psychosocial stress during pregnancy. Am J Obstet Gynecol. 2010;202:61.e61-7.

3. Gavin NI, Gaynes BN, Lohr KN, Meltzer-Brody S, Gartlehner G, Swinson T. Perinatal depression: a systematic review of prevalence and incidence. Obstet Gynecol. 2005;106:1071-83.

4. Bennett HA, Einarson A, Taddio A, Koren G, Einarson TR. Prevalence of depression during pregnancy: systematic review. Obstet Gynecol. 2004;103:698-709.

5. Deputy NP, Sharma AJ, Kim SY, Hinkle SN. Prevalence and characteristics associated with gestational weight gain adequacy. Obstet Gynecol. 2015;125:773-81.

6. Chu SY, Callaghan WM, Bish CL, D'Angelo D. Gestational weight gain by body mass index among US women delivering live births, 2004-2005: fueling future obesity. Am J Obstet Gynecol. 2009;200:271 e271-7.

7. Hartley E, McPhie S, Skouteris H, Fuller-Tyszkiewicz M, Hill B. Psychosocial risk factors for excessive gestational weight gain: a systematic review. Women Birth. 2015;28:e99-e109.

8. Nagl M, Linde K, Stepan H, Kersting A. Obesity and anxiety during pregnancy and postpartum: a systematic review. J Affect Disord. 2015;186:293-305.

9. Kapadia MZ, Gaston A, et al. Psychological antecedents of excess gestational weight gain: a systematic review. BMC Pregnancy Childbirth. 2015;15:107.

10. Catalano PM, Shankar K. Obesity and pregnancy: mechanisms of short term and long term adverse consequences for mother and child. BMJ. 2017;356:j1.

11. Goldstein RF, Abell SK, Ranasinha S, Misso M, Boyle JA, Black $\mathrm{MH}$, et al. Association of gestational weight gain with maternal and infant outcomes: a systematic review and meta-analysis. JAMA. 2017;317:2207-25.

12. Gaillard R, Durmuș B, Hofman A, Mackenbach JP, Steegers EAP, Jaddoe VWV. Risk factors and outcomes of maternal obesity and excessive weight gain during pregnancy. Obesity. 2013;21:104655.

13. Henrichs J, Schenk JJ, et al. Maternal psychological distress and fetal growth trajectories: the Generation R Study. Psychol Med. 2010;40:633-43.

14. Kominiarek MA, Grobman W, et al. Stress during pregnancy and gestational weight gain. J Perinatol. 2018;38:462-7.

15. Shieh $\mathrm{C}, \mathrm{Wu}$ J. Depressive symptoms and obesity/weight gain factors among Black and Hispanic pregnant women. J Community Health Nurs. 2014;31:8-19.

16. Bodnar LM, Wisner KL, Moses-Kolko E, Sit DK, Hanusa BH. Prepregnancy body mass index, gestational weight gain, and the likelihood of major depressive disorder during pregnancy. J Clin Psychiatry. 2009;70:1290-6.

17. Heery E, Kelleher CC, Wall PG, McAuliffe FM. Prediction of gestational weight gain - a biopsychosocial model. Public Health Nutr. 2015;18:1488-98.
18. Kooijman MNKC, van Duijn CM, Duijts L, Franco OH, van IJzendoorn MH, de Jongste JC, et al. The Generation R Study: design and cohort update 2017. Eur J Epidemiol. 2017;31:124364.

19. Jaddoe VW, van Duijn CM, et al. The generation R study: design and cohort update 2012. Eur J Epidemiol. 2012;27:739-56.

20. Derogatis LR, Melisaratos N. The brief symptom inventory: an introductory report. Psychol Med. 1983;13:595-605.

21. Boulet J, Boss MW. Reliability and validity of the Brief Symptom Inventory. J Consult Clin Psychol. 1991;3:433.

22. De Beurs E. Brief symptom inventory. Handleiding. Leiden: Pits Publishers; 2004.

23. De Beurs E. Brief symptom inventory, handleiding addendum. PITS BV: Leiden; 2009.

24. World Health Organization. Obesity: preventing and managing the global epidemic. World Health Organ. 2000.

25. Rasmussen KM, Catalano PM, Yaktine AL. New guidelines for weight gain during pregnancy: what obstetrician/gynecologists should know. Curr Opin Obstet Gynecol. 2009;21:521.

26. Gilmore LA, Redman LM. Weight gain in pregnancy and application of the 2009 IOM guidelines: toward a uniform approach. Obesity. 2015;23:507-11.

27. Rasmussen KM, Yaktine AL. Committee to reexamine IOM pregnancy weight guidelines. Food and nutrition board, board on children, youth and families, Institute of Medicine, National Research Council. Weight gain during pregnancy: reexamining the guidelines. Washington, DC: National Academies Press; 2009.

28. Gaillard R, Steegers EA, de Jongste JC, Hofman A, Jaddoe VW. Tracking of fetal growth characteristics during different trimesters and the risks of adverse birth outcomes. Int J Epidemiol. 2014;43: $1140-53$.

29. Little RJ, Rubin DB. Statistical analysis with missing data. Hoboken: John Wiley \& Sons; 2014.

30. Sterne JA, White IR, et al. Multiple imputation for missing data in epidemiological and clinical research: potential and pitfalls. BMJ. 2009;338:b2393.

31. Webb JB, Siega-Riz AM, Dole N. Psychosocial determinants of adequacy of gestational weight gain. Obesity (Silver Spring). 2009;17:300-9.

32. McPhie S, Skouteris H, Fuller-Tyszkiewicz M, Hill B, Jacka F, O'Neil A. Relationships between mental health symptoms and body mass index in women with and without excessive weight gain during pregnancy. Midwifery. 2015;31:138-46.

33. Ng M, Fleming T, Robinson M, Thomson B, Graetz N, Margono C, et al. Global, regional, and national prevalence of overweight and obesity in children and adults during 1980-2013: a systematic analysis for the global burden of disease study 2013. Lancet. 2014;384: 766-81.

34. Ogden CL, Carroll MD, Flegal KM. Prevalence of obesity in the United States. JAMA. 2014;312:189-90.

35. Molyneaux E, Poston L, Khondoker M, Howard LM. Obesity, antenatal depression, diet and gestational weight gain in a population cohort study. Arch Womens Ment Health. 2016;19:899-907.

36. Pan ML, Tsao HM, et al. Bidirectional association between obstructive sleep apnea and depression: a population-based longitudinal study. Medicine (Baltimore). 2016;95:e4833.

37. Luppino FS, de Wit LM, et al. Overweight, obesity, and depression: a systematic review and meta-analysis of longitudinal studies. Arch Gen Psychiatry. 2010;67:220-9.

38. Hartwig FP, Bowden J, Loret de Mola C, Tovo-Rodrigues L, Davey Smith G, Horta BL. Body mass index and psychiatric disorders: a Mendelian randomization study. Sci Rep. 2016;6:32730.

39. Bogaerts AFL, Devlieger R, Nuyts E, Witters I, Gyselaers W, Van den Bergh BRH. Effects of lifestyle intervention in obese pregnant 
women on gestational weight gain and mental health: a randomized controlled trial. Int J Obes (Lond). 2013;37:814-21.

40. Alder J, Fink N, Bitzer J, Hösli I, Holzgreve W. Depression and anxiety during pregnancy: a risk factor for obstetric, fetal and neonatal outcome? A critical review of the literature. J Matern Fetal Neonatal Med. 2007;20:189-209.
41. Rothman KJ. Epidemiology: an introduction. New York: Oxford University Press; 2002.

Publisher's Note Springer Nature remains neutral with regard to jurisdictional claims in published maps and institutional affiliations. 